\title{
InCEntives, Wages, Employment, AND the Division OF LABOR IN TEAMS
}

August 1, 2013

\begin{abstract}
We develop a theory of incentives, wages, and employment in the context of team production. A central insight is that specialization and division of labor not only improve productivity but also increase effort and the sensitivity of effort to incentives under moral hazard. We show that incentives and employment are complements for the principal when the positive effects of specialization and division of labor outweigh the negative effects of increased idiosyncratic risk and are substitutes otherwise. We provide new characterizations of the partnership, the firm, and the role of the budget-breaker that are quite different from the classical literature.
\end{abstract}

JEL Classifications: D02, D21, D86, L25, M5.

Keywords: budget-breaker, division of labor, employment, endogenous team size, incentives, partnerships, size-wage differential, specialization, teams, wages. 


\section{Introduction}

In this paper we develop a theory of incentives, wages, and employment in a team production context where workers can specialize within a division of labor. ${ }^{1}$ While the positive effects of specialization and division of labor in terms of increased productivity and economic growth have long been recognized, their implications for institutional outcomes under moral hazard have yet to be fully explored. A central insight of the paper is that specialization and division of labor not only improve productivity but under moral hazard and incentive contracting also increase effort and the sensitivity of effort to incentives. The intuition is straightforward: the expected marginal benefit of effort is the piece rate times the expected marginal product of effort which increases with specialization and division of labor. We show that this simple insight in large part determines whether incentives and employment are complements or substitutes for the principal. ${ }^{2}$

Our starting point is the theory of specialization and division of labor in Becker and Murphy (1992), which we extend to allow agents to provide an elastic supply of effort. We first consider the benchmark case where agents' efforts are contractible (i.e., observable and verifiable) and the principal chooses efforts and employment to maximize the expected total surplus. We show that effort and employment are complements for the principal because an increase in employment leads to greater specialization, division of labor, and expected productivity, so the principal increases effort. As we will see, this complementarity between effort and employment can break down under moral hazard. As in Becker and Murphy, the optimal employment level reflects a tradeoff between greater expected productivity and higher transaction costs of employment.

We then embed our extension of the Becker-Murphy model into the classical teams framework due to Alchian and Demsetz (1972) and Holmström (1982). We consider two distinct institutions. The first is the equal-division partnership (EDP) where revenues and transaction costs are divided equally among partners. ${ }^{3}$ The second is the firm with a budget-breaker who offers linear contracts in team output. ${ }^{4}$ In Holmström the partnership is defined by a balanced-budget sharing rule (an

\footnotetext{
${ }^{1}$ Following Becker and Murphy (1992), we define specialization as a task-specific investment in human capital which improves productivity only in that particular task. For a given production process, the division of labor is an assignment of workers to tasks. A more intensive division of labor is when each worker performs fewer tasks.

${ }^{2}$ We say that two endogenous variables are strategic complements if an increase in one optimally implies an increase in the other for given values of the parameters. Likewise, they are substitutes if an increase in one implies a decrease in the other.

${ }^{3}$ Equal-division contracts can be optimal to prevent sabotage as in Bose, Pal, and Sappington (2010), because of inequity aversion in Bartling and von Siemens (2010), and to ensure stability in Levin and Tadelis (2005). Encinosa, Gaynor, and Rebitzer (2007) document the prevalence of equal-division contracts in medical group practices and Lang and Gordon (1995) in law firms in the form of the lock-step system.

${ }^{4}$ The assumptions of linear contracts, additive and normally distributed idiosyncratic shocks, and identical agents
} 
example of which is equal division as in our paper) which provides weak incentives and implements less than first best effort. In his model the role of the budget-breaker is to address the free-rider problem by replacing weak balanced-budget incentives with stronger incentives that implement the first best (or arbitrarily close in the case of uncertainty). This characterization contradicts the stylized fact that incentives are weak in firms (see Prendergast (1999)). Absent a budget-breaker, Alchian and Demsetz speculate that partnerships will tend to be small to control free-riding. ${ }^{5}$ Since Alchian and Demsetz do not provide a formal model and employment is exogenous in Holmström, the present paper appears to be the first to systematically examine these issues within a unified theoretical framework.

In our model an EDP has several distinctive characteristics. First, the equal division of revenue creates a $1 / n$ problem with respect to effort because incentives decline with team size $n$. This is a specific instance of the more general point in Holmström about the weak nature of balanced-budget incentives. The equal division of revenue also creates a $1 / n$ problem with respect to employment as emphasized in Levin and Tadelis (2005): the representative partner only receives $1 / n$ of the expected return from the marginal hire which biases an EDP towards under-employment. Second, the fact that the transaction costs of employment are divided equally among partners implies another albeit positive $1 / n$ effect on employment: the representative partner only considers his share of transaction costs whereas the principal in the benchmark case and the budget-breaker in the firm bear the full costs. Finally, in an EDP there is a unique relationship between employment and risk. For simplicity we follow Auriol, Friebel, and Pechlivanos (1999, 2002) in assuming that risk increases with employment because of additional idiosyncratic risk. ${ }^{6}$ As in Lang and Gordon (1995) and Levin and Tadelis (2005), an EDP chooses employment to maximize the expected payoff of the representative partner, who only considers his own individual risk premium in contrast to the budget-breaker in the firm who considers the aggregate risk premium. We use a simple example to show that an EDP is not necessarily small as indicated by Alchian and Demsetz; indeed, we show that an EDP can be the same size as in the benchmark case with contractible effort.

with exponential utility are made for tractability but constitute an important limitation of the model. As is wellknown (see Bolton and Dewatripont (2005, Section 4.3)), linear contracts are generally suboptimal under these conditions. Given the structure of our model, in particular the additive nature of the production function, there is reason to believe that the classic rationale in Holmström and Milgrom (1987) which applies for a single agent also applies here. Under limited liability, Bose, Pal, and Sappington (2011) show that the optimal linear contract achieves at least $90 \%$ of the expected profit of the optimal nonlinear contract. A linear contract will therefore be optimal when transaction costs are sufficiently increasing in contractual complexity.

${ }^{5}$ A more recent literature including Rasmusen (1987), Legros and Matsushima (1991), Legros and Matthews (1993), Garicano and Santos (2004), and Levin and Tadelis (2005) identifies conditions under which the partnership achieves the first best or is more efficient than some alternative institution.

${ }^{6}$ Another approach would be to explicitly model the monitoring technology as in Rasmusen and Zenger (1990), where monitoring costs increase with firm size. 
We then turn to the firm. We first consider the agents' non-cooperative choice of efforts given the set of contracts and employment level. Given incentives, we show that optimal effort and the sensitivity of effort to incentives are both increasing in employment. An increase in employment also improves productivity through greater specialization and division of labor and the combination of these two effects on effort and productivity tends to make incentives and employment complements for the budget-breaker. On the other hand, an increase in employment increases idiosyncratic risk which tends to make them substitutes. Whether incentives and employment are ultimately complements or substitutes is therefore determined by the stronger of these two classes of effects. We discuss some empirical evidence which suggests that they are in fact substitutes.

In the standard linear model of Holmström and Milgrom $(1987,1991)$ incentives are decreasing in risk because of the classical risk-reward tradeoff. In our model with endogenous employment the strength of the relationship between incentives and risk depends on whether incentives and employment are complements or substitutes. In particular, we show that the relationship is weaker than in the standard model when incentives and employment are substitutes (indeed, we provide an example where incentives are independent of risk) and stronger when they are complements. This result provides an unexpected link between the aforementioned evidence that incentives and employment are substitutes and the mixed nature of the evidence on the risk-reward tradeoff as surveyed in Prendergast (1999).

We also show that the expected wage is increasing in the size of the firm as measured by employment. According to Brown and Medoff (1989, p. 1028), "the size-wage differential is one of the key differentials observed in labor markets." Brown and Medoff show that differences in worker quality can explain about one-half of the size-wage differential but find little or no evidence in support of any of the other conventional explanations. In our model larger firms are characterized by greater idiosyncratic risk and higher effort due to greater expected productivity. The fact that larger firms pay higher wages therefore follows from the participation constraint.

Finally, we compare the firm to the EDP in the same example we used to compare the EDP to the benchmark case. We obtain the classical characterization of these two institutions when risk and transaction costs are low: the EDP is smaller with weaker incentives and less effort compared to the firm and the role of the budget-breaker is to provide stronger incentives to increase effort as in Holmström. In contrast, we obtain the opposite characterization when risk and transaction costs are high: the EDP is larger with stronger incentives and higher effort than the firm and the role of the budget-breaker is to downsize the EDP and offer weaker incentives to reduce the agents' exposure to risk. In this case employment is higher in an EDP than in the firm because 
the representative partner in an EDP only considers his individual (as opposed to aggregate) risk premium and only bears his share (rather than the total) of the transaction costs of employment. Weak incentives in firms imply higher effort in an EDP. Consistent with these predictions, Monteiro and Stewart (2012) document that in Portugal the average labor-managed firm is larger than the average capitalist firm. According to Venn (2009), Portugal is one of the strictest OECD countries in terms of employment protection (e.g., difficulty of dismissal and regulation of temporary contracts) which suggests relatively high employment transaction costs.

Our focus on incentives, employment, and the division of labor is shared by the literature on job design, including Holmström and Milgrom (1991), Itoh (1994), Schmitz (2005), and Schöttner (2008). The central issue in this literature is whether a principal should, for example, hire one agent to perform two tasks or two agents who can specialize (to use their terminology). The answer generally revolves around incentive considerations. Despite these shared concerns the questions we ask in this paper are different and in comparison the employment decision in our model is richer while the job design aspect is correspondingly cruder (see the appendix to this paper).

As far as we know there are only two papers in the teams literature where employment is endogenous. Auriol et al. (1999) derive an expression for the optimal incentive similar to ours where incentives and employment are substitutes. Liang, Rajan, and Ray (2008) assume the variance of the group performance measure is $G(n) \sigma^{2}$, where $G$ is a reduced-form which captures the idea that monitoring becomes more difficult as team size $n$ grows. In their model incentives and employment are substitutes and the risk-reward tradeoff is weaker than in the standard model. Under certain conditions on the reduced-form $G$ the optimal incentive is independent of all the parameters including risk $\sigma^{2}$. In our model with specialization and division of labor incentives and employment can be complements or substitutes and the relationship between incentives and risk correspondingly stronger or weaker than in the standard model.

The plan for the rest of the paper is as follows. In the next section we characterize the benchmark case with contractible efforts. We consider moral hazard in section 3, the EDP in section 4, and the firm in section 5. Section 6 shows how the predictions of the model can be tested using crosssectional data and discusses the available evidence. Section 7 concludes. All proofs are in the appendix. 


\section{Contractible Effort}

We consider one principal and a population of size $\bar{n} \geq 1$ of identical agents. Let $N=\{0,1,2, \ldots, \bar{n}\}$ and $n \in N$ be the number of agents employed by the principal. Note that $n$ is restricted to be a nonnegative integer although in examples we will sometimes ignore the integer constraint. The output of agent $i$ when employed is

$$
Q_{i}=\phi(n) e_{i}+\epsilon_{i}
$$

where $\phi(0)=0$ and $\phi$ is strictly increasing in $n \in N, e_{i}$ is the effort of agent $i$, and $\epsilon_{i}$ is an i.i.d. normally distributed idiosyncratic shock with mean zero and variance $\sigma^{2}{ }^{7}$ We assume $\epsilon_{i}$ is either unrealized or unobservable to all parties prior to contracting. Examples include shocks to human capital and other individual characteristics related to productivity. Output is given by ${ }^{8}$

$$
Q=\sum_{i} Q_{i}=\phi(n) \sum_{i} e_{i}+\sum_{i} \epsilon_{i}
$$

In the Appendix we show how (2) can be derived from more primitive assumptions about the production process in a framework similar to that in Becker and Murphy (1992). As in their paper the central theme is that an increase in employment $n$ leads to greater specialization and division of labor and hence higher expected productivity $\phi$. The prediction that larger teams are more specialized with a more intensive division of labor is a consistent empirical finding in the management literature; see Carter and Keon (1986) for a survey. The assumption that $\phi$ is strictly increasing in $n$ implies that expected output per worker is increasing in employment for any symmetric effort profile. This is consistent with the traditional notion that specialization and division of labor imply increasing returns to scale.

We assume that hiring new workers involves transaction costs $k c(n)$, where $c$ is strictly increasing in $n \in N$ and $k>0$ is a parameter which takes values in $K$, an interval in the positive reals. Examples include healthcare, legal, regulatory, and basic training costs, new machinery, increases in plant size and other physical costs, as well as greater coordination costs (Becker and Murphy emphasize the latter). Let

$$
K^{-1}=\left\{k^{-1} \mid k \in K\right\}
$$

\footnotetext{
${ }^{7}$ If $x=\left(x_{i}\right)$ and $x^{\prime}=\left(x_{i}^{\prime}\right)$ are vectors in Euclidean space $\mathbb{R}^{m}$ then $x \geq x^{\prime}$ iff $x_{i} \geq x_{i}^{\prime}$ for all $1 \leq i \leq m$. We write $x>x^{\prime}$ if $x \geq x^{\prime}$ and $x \neq x^{\prime}$. If $A \subseteq \mathbb{R}^{m}$ and $f: A \rightarrow \mathbb{R}$ then $f$ is increasing if $x>x^{\prime}$ implies $f(x) \geq f\left(x^{\prime}\right)$ and strictly increasing when the inequality is strict.

${ }^{8}$ In (2) the agents' efforts are perfect substitutes. In the working paper version, available at SSRN, we consider a stochastic Leontief production function where efforts are perfect complements. The main results are qualitatively similar.
} 
and $p \in P$ be the price of output, where $P$ is also an interval in the positive reals.

In this section we consider the benchmark case where effort is contractible. The principal's problem is therefore to choose team size $n$ and efforts $\left\{e_{i}\right\}$ to maximize the expected total surplus

$$
p \phi(n) \sum_{i} e_{i}-(1 / 2) \sum_{i} e_{i}^{2}-k c(n)
$$

subject to the constraints $n \in N$ and $e_{i} \geq 0$ for all $i$. The first term in (4) is expected revenue and the second is the sum of quadratic effort costs. Given $n \geq 1$, the optimal effort of agent $i$ is

$$
e_{i}=p \phi(n)
$$

It follows that effort and employment are strategic complements for the principal in the sense that an increase in one optimally implies an increase in the other for given values of the parameters. Intuitively, an increase in team size leads to greater specialization and division of labor which improves expected productivity, so the principal increases effort. This result is new because Becker and Murphy assume that each agent supplies one unit of effort inelastically. In subsequent sections we will show that this complementarity between effort and employment is weakened by moral hazard.

Substituting (5) into (4),

$$
V\left(n, p, k^{-1}\right)=(1 / 2) n p^{2} \phi(n)^{2}-\left(k^{-1}\right)^{-1} c(n),
$$

which we have written in terms of $k^{-1}$ rather than $k$ for reasons that will be clear momentarily. The principal chooses $n$ to maximize (6) subject to the constraint $n \in N$. In this paper existence of optimal solutions is never an issue because $N$ is a finite set.

To state our results requires some definitions from lattice programming and the theory of supermodular games. Let $X \subseteq \mathbb{R}^{m}$ and for all $x, x^{\prime} \in \mathbb{R}^{m 9}$

$$
x \vee_{X} x^{\prime}=\sup _{X}\left\{x, x^{\prime}\right\} \text { and } x \wedge_{X} x^{\prime}=\inf _{X}\left\{x, x^{\prime}\right\}
$$

$X$ is a lattice if $x \vee_{X} x^{\prime}$ and $x \wedge_{X} x^{\prime}$ exist for all $x, x^{\prime} \in X . X$ is a sublattice of $\mathbb{R}^{m}$ if $x \vee_{\mathbb{R}^{m}} x^{\prime} \in X$ and $x \wedge_{\mathbb{R}^{m}} x^{\prime} \in X$ for all $x, x^{\prime} \in X$. An example is $N$, which is a lattice and a sublattice of $\mathbb{R}$. If

\footnotetext{
${ }^{9}$ Let $A, X \subseteq \mathbb{R}^{m}$. An upper bound for $A$ in $X$ is an $x \in X$ such that $x \geq a$ for all $a \in A$. If $x \leq x^{\prime}$ for any other upper bound $\overline{x^{\prime}}$ for $A$ in $X$ then $x$ is the least upper bound or supremum of $\bar{A}$ in $X$ denoted $x=\sup _{X} A$. The infimum of $A$ in $X$ is defined similarly as the greatest lower bound of $A$ in $X$.
} 
$X$ is a lattice and $T \subseteq \mathbb{R}^{l}$ the function $h(x, t)$ has strictly increasing differences on $X \times T$ if $t>t^{\prime}$ implies $h(x, t)-h\left(x, t^{\prime}\right)$ is strictly increasing in $x$. If $X$ and $T$ are open intervals and $h$ is twice continuously differentiable this is equivalent to $\partial^{2} h / \partial x \partial t>0$ on $X \times T$. Intuitively, an increase in $t$ raises the marginal return to increasing $x$. A correspondence $\Gamma: T \rightarrow X$ is strongly increasing if $t>t^{\prime}$ implies $x \geq x^{\prime}$ for all $x \in \Gamma(t)$ and $x^{\prime} \in \Gamma\left(t^{\prime}\right)$. An increase in $t$ therefore shifts the set $\Gamma(t)$ upwards. The definition for strongly decreasing is similar. For comprehensive treatments of these concepts see Topkis (1998) and Vives (1999).

Alternatively, we could use classical comparative statics techniques if we allowed $n$ to be a continuous variable and the principal's choice set to be the interval $[0, \bar{n}]$ rather than the discrete set $N$. We use lattice programming techniques because they are analytically more convenient, can easily handle potential corner solutions at $n=0$ or $n=\bar{n}$, and quasi-concavity can fail under increasing returns. Note that the lattice programming techniques we are using treat discrete and continuous choice sets equally; i.e., we can impose the integer constraint on the employment level $n$ at no extra analytical cost.

Let

$$
\begin{aligned}
& n_{c}\left(p, k^{-1}\right)=\arg \max _{n \in N} V\left(n, p, k^{-1}\right) \\
& e_{c}\left(p, k^{-1}\right)=\left\{p \phi(n) \mid n \in n_{c}\left(p, k^{-1}\right)\right\},
\end{aligned}
$$

the sets of optimal employment and effort levels, respectively. In general these are correspondences rather than functions because we have not made any assumptions which would ensure a unique optimal employment level.

\section{Proposition 1}

(i) Effort and employment are complements for the principal when effort is contractible.

(ii) $V$ in (6) has strictly increasing differences on $N \times\left(P \times K^{-1}\right)$.

(iii) $n_{c}$ and $e_{c}$ are strongly increasing in $\left(p, k^{-1}\right)$.

The first result records the fact discussed previously. The second one states that the expected total surplus (6) has strictly increasing differences in $n$ and $\left(p, k^{-1}\right)$, which means that an increase in price $p$ or a decrease in the transaction cost parameter $k$ raises the expected marginal return to increasing employment. The intuition for an increase in $p$ revolves around the term $n \phi^{2}$ in (6) which will also appear in the context of the firm. First, an increase in $p$ raises the expected marginal 
return to increasing output. An increase in employment would serve this purpose simply because there are more workers (the term $n$ ) and it increases productivity $\phi$. Second, an increase in $p$ increases the sensitivity of optimal effort (5) to an increase in employment and this effect combined with the increase in productivity are reflected in the term $\phi^{2}$. Strictly increasing differences imply the monotone comparative statics in (iii), which states that an increase in the parameters $\left(p, k^{-1}\right)$ shifts the set of optimal employment levels upwards. Since employment and effort are complements, this produces a corresponding upward shift in the set of optimal effort levels. In the special case where the optimal employment level is unique, $n_{c}$ and $e_{c}$ are increasing functions in $p$ and $k^{-1}$.

The following simple example illustrates these comparative statics and will be used later to compare institutions. ${ }^{10}$

Example 1 If $\phi(n)=A n^{1 / 2}$ and $c(n)=(1 / 4) n^{4}$ the benchmark problem has a unique closed-form solution

$$
n_{c}=\frac{A p}{k^{1 / 2}} \quad \text { and } \quad e_{c}=\frac{(A p)^{3 / 2}}{k^{1 / 4}}
$$

ignoring the integer constraint for employment.

\section{Moral Hazard}

We now move beyond the contractible effort benchmark in Becker and Murphy to the case of moral hazard. Following the classical teams literature we focus on two distinct institutions. The first is the firm with a budget-breaker who offers a linear contract $I_{i}=\alpha_{i}+\beta_{i} Q$ to agent $i$, where $I_{i}$ is the total wage, $\alpha_{i}$ is a lump-sum payment, and $\beta_{i}$ the piece rate or incentive on team output $Q$. The second institution is an EDP where revenue and transaction costs are divided equally among partners and there are no lump-sum transfers. This is the special case where $\alpha_{i}=0$ and $\beta_{i}=p / n$ for all $i{ }^{11}$ We refer to $p / n$ as the equal-division incentive because it is the implicit incentive implied by equal division of revenue. Note that this is a balanced-budget sharing rule as defined in Holmström (1982).

The agents' utility functions are negative exponential

$$
-\exp \left\{-r\left[I_{i}-(1 / 2) e_{i}^{2}\right]\right\}
$$

\footnotetext{
${ }^{10}$ Assume $N=\infty$ in all examples throughout the paper.

${ }^{11}$ Although the contract offered by an EDP is one element in the set of contracts open to the budget-breaker, an EDP is not a special case of the firm because their objective functions are different: an EDP maximizes the expected payoff of the representative partner while the firm maximizes expected profit.
} 
where $r>0$ is the coefficient of absolute risk aversion (CARA) and the quadratic term is the cost of effort. The corresponding certainty equivalent payoff is given by ${ }^{12}$

$$
U_{i}=\alpha_{i}+\beta_{i} \phi(n) \sum_{i} e_{i}-(1 / 2) e_{i}^{2}-(1 / 2) \beta_{i}^{2} n v
$$

where $v=r \sigma^{2}$. The first two terms on the right-hand side constitute the expected wage as a function of expected output. The final term is the risk premium, the disutility of risk imposed by the contract, which links the agent's income to stochastic output. The risk premium is increasing in the CARA $r$, the incentive $\beta_{i}$ (because stronger incentives tie income more closely to stochastic output), the variance $\sigma^{2}$ of the idiosyncratic shocks, and employment $n$ (because more workers imply more idiosyncratic risk). We refer to $v$ as subjective risk because it is the objective risk $\sigma^{2}$ imposed by each additional worker scaled by the CARA.

The timing of the game is as follows. (i) The budget-breaker chooses the number $n$ of workers and the contracts $\left\{\left(\alpha_{i}, \beta_{i}\right)\right\}$. In an EDP, the sole choice variable is partnership size $n$ because there are no transfers and the equal-division incentive is an intrinsic institutional feature. (ii) Agents who receive offers decide whether or not to accept them. We assume an outside option of zero and that indifferent agents accept. (iii) Those who accept choose their efforts non-cooperatively. (iv) Output is realized and the contract is executed. The budget-breaker chooses $n$ to maximize her expected profits. As in Lang and Gordon (1995) and Levin and Tadelis (2005), an EDP chooses $n$ to maximize the expected payoff of the representative partner.

Given employment $n \geq 1$ and the set of contracts the optimal effort of agent $i$ in stage (iii) is

$$
e_{i}=\beta_{i} \phi(n)
$$

Since $\phi$ is strictly increasing the principal can increase effort with either an increase in incentives $\beta_{i}$ and/or an increase in employment $n$. Intuitively, an increase in employment leads to greater specialization and division of labor and higher expected productivity. This raises the expected marginal benefit of effort because an additional unit of effort increases expected incentive pay more when productivity is higher. This is one of the central insights of the paper: in addition to their classical effects on productivity, specialization and division of labor also have a direct and positive impact on effort in a moral hazard context with incentive contracting (note that there is no effect on effort with zero incentives). The fact that an increase in employment increases the sensitivity

\footnotetext{
${ }^{12}$ Bolton and Dewatripont (2005, p. 137) provide a detailed derivation.
} 
of effort to incentives tends to make incentives and employment complements for the principal. As we will see, however, there are other aspects of the model which tend to make them substitutes.

In an EDP $\beta_{i}=p / n$ and (13) becomes

$$
e_{i}=\frac{p \phi(n)}{n}
$$

An increase in employment improves productivity $\phi$ through greater specialization and division of labor but it also dilutes the equal-division incentive $p / n$ (the $1 / n$ effect with respect to effort). If the first effect dominates so that $\phi(n) / n$ is increasing in $n$ then optimal effort is increasing in employment and effort and employment are complements in an EDP. We obtain the opposite result when the second effect dominates and $\phi(n) / n$ is decreasing. In comparison with the benchmark case, moral hazard breaks the automatic complementarity between effort and employment. Furthermore, optimal EDP effort (14) is less than optimal benchmark effort (5) when team size is exogenous, the same in both contexts, and $n \geq 2$. This is the effect of balanced-budget sharing rules identified by Holmström (1982).

\section{Equal Division Partnerships}

We first consider the EDP. Substituting $\alpha_{i}=0$ and $\beta_{i}=p / n$ into (12) and subtracting the individual's share $k c(n) / n$ of transaction costs, the expected payoff of the representative partner is

$$
\frac{p}{n} \phi(n) \sum_{i} e_{i}-(1 / 2) e_{i}^{2}-(1 / 2)\left(\frac{p}{n}\right)^{2} n v-k \frac{c(n)}{n} .
$$

Note that an increase in employment $n$ reduces the risk premium of the representative partner by diluting the equal-division incentive. Substituting optimal effort ${ }^{13}(14)$,

$$
U^{p}(n, p, k, v)=p^{2}\left[f(n)-\frac{v}{2 n}\right]-k \frac{c(n)}{n},
$$

where

$$
f(n) \equiv \frac{\phi(n)^{2}}{n}\left(1-\frac{1}{2 n}\right)
$$

\footnotetext{
${ }^{13}$ From now on we define functions in terms of $k$ and $v$ rather than $k^{-1}$ and $v^{-1}$.
} 
Define $U^{p}=0$ when $n=0$. An EDP chooses the number $n$ of partners to maximize (16) subject to the constraint $n \in N$. Let

$$
\begin{aligned}
& n_{p}(p, k, v)=\arg \max _{n \in N} U^{p}(n, p, k, v) \\
& e_{p}(p, k, v)=\left\{\frac{p \phi(n)}{n} \mid n \in n_{p}(p, k, v)\right\},
\end{aligned}
$$

the optimal EDP employment and effort correspondences, respectively.

Proposition 2 If $f(n)$ and $c(n) / n$ are strictly increasing in $n$ for all $n \geq 1$ then (i) $U^{p}$ has strictly increasing differences in $n$ and $\left(p, k^{-1}, v\right)$ for all $n \geq 1$ and (ii) $n_{p}$ is strongly increasing for all $\left(p, k^{-1}, v\right)$ such that $0 \notin n_{p}(p, k, v)$.

Since the objective function $U^{p}$ in (16) is not well-behaved at $n=0$ the results are stated in terms of positive employment levels. The first result states that an increase in $\left(p, k^{-1}, v\right)$ raises the expected marginal benefit of increasing employment starting from any positive employment level. We first consider the effects of an increase in price $p$. From (15), an increase in $p$ raises the risk premium of the representative partner through an increase in the equal-division incentive $p / n$. This makes an increase in employment $n$ desirable to reduce the risk premium. An increase in $p$ also raises the expected marginal benefit of increasing output. An increase in employment increases expected output by improving productivity $\phi$ and increasing aggregate optimal effort $p \phi(n)$. On the other hand, an increase in employment would also dilute the equal-division incentive. If $\phi$ is sufficiently increasing in $n$ in the sense that $f$ is strictly increasing then the combined effect is positive and the EDP increases employment. An increase in subjective risk $v$ induces an increase in employment to counter the corresponding increase in the risk premium. Finally, an increase in the transaction cost parameter $k$ reduces employment if the representative partner's share $c(n) / n$ of transaction costs is increasing in $n$. The second result states that an increase in any one or combination of the parameters leads to an upward shift in the set of optimal employment levels provided that zero employment is not optimal initially.

Given these results for optimal employment $n_{p}$, the comparative statics for optimal effort $e_{p}$ depend on whether (14) is increasing or decreasing in employment.

Proposition 3 Assume $c(n) / n$ is strictly increasing for all $n \geq 1$ and zero employment is not optimal.

(i) If $\phi(n) / n$ is strictly increasing for all $n \geq 1$ then $n_{p}$ and $e_{p}$ are both strongly increasing in $\left(p, k^{-1}, v\right)$. 
(ii) If $\phi(n) / n$ is strictly decreasing but $f(n)$ is strictly increasing for all $n \geq 1$ then $e_{p}$ is strongly decreasing in $\left(k^{-1}, v\right)$.

The first result is straightforward. If $\phi / n$ is strictly increasing then $f(n)$ is strictly increasing and the previous proposition applies. Since optimal effort (14) is strictly increasing in $n$ the comparative statics for $e_{p}$ follow from those for $n_{p}$. In the second result $\phi / n$ is strictly decreasing so (14) is strictly decreasing in employment $n$. In this case $e_{p}$ is strongly decreasing in $\left(k^{-1}, v\right)$ because optimal employment $n_{p}$ is strongly increasing in those parameters. The comparative static for $p$, however, is ambiguous: an increase in price increases (14) directly but also reduces it indirectly through an increase in employment. The following example illustrates result (ii); in this particular example $e_{p}$ is strictly increasing in $p$.

Example 2 If $\phi(n)=n^{1 / 2}$ and $c(n)=(1 / 4) n^{4}$ then

(i) the EDP problem has a unique closed-form solution

$$
e_{p}=\left[\frac{3 p^{6} k}{2(1+v)}\right]^{1 / 8} \quad \text { and } \quad n_{p}=\left[\frac{2 p^{2}(1+v)}{3 k}\right]^{1 / 4}
$$

ignoring the integer constraint.

(ii) In comparison with the benchmark case in Example 1:

$$
\begin{aligned}
U^{p} \gtreqless 0 & \Longleftrightarrow A^{8} p^{2} \gtreqless(2 / 3)^{3} k\left(A^{2}+v\right)^{3} \\
n_{c} \gtreqless n_{p} & \Longleftrightarrow A^{4} p^{2} \gtreqless(2 / 3) k\left(A^{2}+v\right) \\
e_{c} \gtreqless e_{p} & \Longleftrightarrow A^{4} p^{6} \gtreqless \frac{3 k^{3}}{2\left(A^{2}+v\right)} .
\end{aligned}
$$

The second result compares optimal EDP effort $e_{p}$ and employment $n_{p}$ to their counterparts $e_{c}$ and $n_{c}$ in the benchmark case with contractible effort. To identify sources of potential differences we compare the objective functions in (4) and (15). First, there is the $1 / n$ effect on revenue: in an EDP the representative partner only receives $1 / n$ of the expected revenue generated by his effort and the marginal hire which tends to make $e_{p}$ and $n_{p}$ less than $e_{c}$ and $n_{c}$. Second, an increase in employment reduces the risk premium of the representative partner which tends to make $n_{p}$ higher than $n_{c}$. Third and finally there is the $1 / n$ effect on transaction costs: while the principal in the benchmark case bears the full transaction costs of employment, the representative partner only considers his share $k c(n) / n$ which also tends to make $n_{p}$ higher than $n_{c}$. From (ii) of the example 
we observe that $e_{p}$ and $n_{p}$ are high relative to $e_{c}$ and $n_{c}$ when $A$ and $p$ are low, which reduce the importance of the first effect, and when $k$ and $v$ are high, which increase the importance of the second and third.

What the example shows is that an EDP can be the same size as in the benchmark case. For example, $n_{c}=2$ and $n_{p} \approx 1.5$ when $A=1 / 3, p=6, k=1$, and $v=1 / 10$. Since the expected payoff of the representative partner is $1 / 10$ at $n=2$ and $-1 / 20$ at $n=1$, we have $n_{c}=n_{p}=2$ when the integer constraint is imposed. This contradicts the characterization of the partnership in Alchian and Demsetz (1972, p. 790) that "these organizations will be small to prevent an excessive dilution of efforts through shirking" although the reference of comparison (first best or capitalist firm) is unclear. While our example suggests that effort and employment can be higher in an EDP than in the benchmark case this only occurs when $n_{c}$ and $n_{p}$ are between zero and one and therefore does not survive the integer constraint.

\section{The Firm}

We now introduce a budget-breaker. The firm's profit equals revenue less wages and transaction costs

$$
p Q-\sum_{i} I_{i}-k c(n)
$$

The budget-breaker's problem is to choose the number $n$ of workers and the contracts $\left\{\left(\alpha_{i}, \beta_{i}\right)\right\}$ to maximize expected profit

$$
p \phi(n) \sum_{i} e_{i}-\sum_{i}\left[\alpha_{i}+\beta_{i} \phi(n) \sum_{i} e_{i}\right]-k c(n)
$$

subject to $n \in N$ and the incentive compatibility (13) and participation constraints $U_{i} \geq 0$.

To state our main comparative statics result, we need to write expected profit as a function of employment $n$ only. We first note that the budget-breaker chooses the lump sums $\alpha_{i}$ to make the participation constraints bind. Substituting $U_{i}=0$ into (25),

$$
p \phi(n) \sum_{i} e_{i}-(1 / 2) \sum_{i} e_{i}^{2}-(1 / 2) n v \sum_{i} \beta_{i}^{2}-k c(n) .
$$

Substituting (13),

$$
p \phi(n)^{2} \sum_{i} \beta_{i}-(1 / 2)\left[\phi(n)^{2}+n v\right] \sum_{i} \beta_{i}^{2}-k c(n) .
$$


The optimal incentive is therefore

$$
\beta=\frac{p \phi(n)^{2}}{\phi(n)^{2}+n v}=p g(n, v)
$$

where

$$
g(n, v) \equiv \frac{\phi(n)^{2}}{\phi(n)^{2}+n v}
$$

We assume $g$ is well-defined at $n=0$ (see the examples that follow).

The expression in (28) already reveals some important comparative statics information. If $g$ is decreasing in $n$ then employment and incentives are substitutes for the budget-breaker in the sense that an increase in $n$ implies a decrease in $\beta$ for given values of the parameters. This can also be expressed as: specialization and division of labor are substitutes for incentives because specialization and division of labor increase with employment. Alternatively, employment and incentives are complements when $g$ is increasing. If $n \geq 1$ then $\phi>0$ and

$$
g(n, v)=\frac{1}{1+\frac{n v}{\phi(n)^{2}}}
$$

so the behavior of $g$ is determined by $\phi^{2} / n$. To interpret this, note that $n$ multiplies subjective risk $v$ and therefore captures the effect of employment on the aggregate risk premium. Intuitively, an increase in employment increases productivity $\phi$ and the sensitivity $\phi$ of effort to incentives (hence the term $\phi^{2}$ ) and therefore raises the expected marginal return to increasing incentives. On the other hand, an increase in employment also increases the aggregate risk premium through additional idiosyncratic risk. The relative magnitudes of these effects determine whether employment and incentives are complements or substitutes.

Substituting (28) into (13) we obtain optimal effort in the firm

$$
e=p g(n, v) \phi(n)
$$

Finally, substituting (28) into (27),

$$
\Pi(n, p, k, v)=(1 / 2) n p^{2} \phi(n)^{2} g(n, v)-k c(n),
$$

which is solely a function of $n$. Comparing (31) and (32) with their counterparts (5) and (6) in the benchmark case, we observe that $g$ completely captures the effects of incentive contracting under moral hazard, which include the aggregate risk premium as well as the impact of incentives on effort. 
Note that if $g$ is decreasing in $n$ then moral hazard weakens the complementarity between effort and employment which existed in the benchmark case. The budget-breaker's optimal employment correspondence is

$$
n_{b}(p, k, v)=\arg \max _{n \in N} \Pi(n, p, k, v) .
$$

From (28) and (31), the optimal incentive and effort correspondences are

$$
\begin{aligned}
& \beta_{b}(p, k, v)=\left\{p g(n, v) \mid n \in n_{b}(p, k, v)\right\} \\
& e_{b}(p, k, v)=\left\{p g(n, v) \phi(n) \mid n \in n_{b}(p, k, v)\right\} .
\end{aligned}
$$

\section{Theorem 1}

(i) The budget-breaker's expected profit $\Pi$ in (32) has strictly increasing differences in $n$ and $\left(p, k^{-1}, v^{-1}\right)$.

(ii) The optimal employment correspondence $n_{b}$ is strongly increasing in $\left(p, k^{-1}, v^{-1}\right)$.

The first result states that an increase in $\left(p, k^{-1}, v^{-1}\right)$ raises the expected marginal return to increasing employment. We first consider an increase in the price $p$. The term $n \phi^{2} g$ in expected profit (32) consists of the term $n \phi^{2}$ which appeared in the benchmark case [see (6)] and the moral hazard term $g$. Holding $g$ fixed, an increase in $p$ raises the expected marginal return to increasing employment as discussed previously in the benchmark case. The effect of moral hazard, however, is ambiguous and depends on whether $g$ is increasing or decreasing in $n$. For example, if $\phi^{2} / n$ is decreasing in $n$ then an increase in employment would have a larger impact on idiosyncratic risk and the risk premium than on productivity and the sensitivity of effort to incentives. In that case, moral hazard reduces the expected marginal return to increasing employment. In the proof (see the appendix) we show that the positive benchmark effect $n \phi^{2}$ outweighs any potential negative effect from $g$ so an increase in $p$ raises the expected marginal return to increasing employment. The comparative statics of an increase in the transaction cost parameter $k$ are clear and exactly the same as in the benchmark case.

Now consider the effect of an increase in $v^{-1}$ or equivalently a decrease in subjective risk $v$ on the expected marginal return to increasing employment. As before, the latter includes the benchmark

effects $n \phi^{2}$ but now we must account for the impact of the reduction in $v$ on the moral hazard term $g$. Again this is ambiguous and changes in $v$ generally produce non-monotonicities in the marginal return from employment from a purely moral hazard perspective: it can be optimal to increase employment following a decrease in $v$ because the cost of employment in terms of idiosyncratic risk 
is lower but it can also be optimal to complement the decrease in $v$ with a decrease in employment to further reduce the aggregate risk premium. ${ }^{14}$ In the proof we show that once again the positive benchmark effects $n \phi^{2}$ dominate and the principal prefers to increase employment following a reduction in $v$.

Given these monotone comparative statics for employment, the comparative statics for effort and incentives follow from (28) and (31) as discussed previously. We now summarize the two main benchmarks, where employment and incentives are either complements or substitutes.

\section{Corollary 1}

(i) If $g(n, v)$ is increasing in $n$ then incentives and employment are complements and $\beta_{b}, e_{b}$, and $n_{b}$ are all strongly increasing in $\left(p, k^{-1}, v^{-1}\right)$.

(ii) If $g(n, v)$ is decreasing but $\phi(n) g(n, v)$ is increasing in $n$ then employment and incentives are substitutes, $e_{b}$ and $n_{b}$ are strongly increasing in $\left(p, k^{-1}, v^{-1}\right)$, and $\beta_{b}$ is strongly decreasing in $k^{-1}$.

The first result is straightforward. If $g$ is increasing in $n$ then incentives and employment are complements. An increase in $\left(p, k^{-1}, v^{-1}\right)$ increases employment which in turn increases incentives (28) and effort (31). An increase in the price $p$ or a decrease in subjective risk $v$ also have direct and positive effects on incentives and effort in addition to their indirect effects operating through employment. The following example illustrates these comparative statics.

Example 3 If $\phi(n)=n$ and $c(n)=(1 / 4) n^{4}$ then

$$
n_{b}=\frac{3 p^{2}-4 k v+p \sqrt{9 p^{2}+8 k v}}{4 k} \quad \text { and } \quad \beta_{b}=\frac{\left(3 p^{2}-4 k v+p \sqrt{9 p^{2}+8 k v}\right)^{2}}{4 k\left(3 p+\sqrt{9 p^{2}+8 k v}\right)} \text {. }
$$

A novel aspect of the model is that parameters normally associated with the employment decision such as the transaction cost parameter $k$ also have implications for incentive contracting. For example, labor market reforms that reduce the transaction costs of hiring new employees will not only lead to lower unemployment but also stronger incentives when incentives and employment are complements. This intermingling of labor market and contractual parameters is clearly evident in the above example where the effect of subjective risk $v$ on the endogenous variables is stronger when $k$ is larger.

\footnotetext{
${ }^{14}$ For example, $\partial g / \partial v$ is non-monotonic in $n$ when $\phi(n)=n$.
} 
We now turn to Corollary 1(ii). If $\phi g$ is increasing in $n$ then effort (31) and employment are complements so the comparative statics for effort follow directly from those for employment as before. If $g$ is decreasing in $n$ then incentives and employment are substitutes so a reduction in $k$ (or equivalently an increase in $k^{-1}$ ) which increases employment will also reduce incentives. The comparative statics for price $p$ and subjective risk $v$ are, however, ambiguous. Recall that in the standard linear model with one agent the optimal incentive is

$$
\beta=\frac{p}{1+v}
$$

(see Bolton and Dewatripont (2005, p. 139)). Note that (28) reduces to (37) when $n=1$ and $\phi(1)=1$. In the standard case (37) incentives are decreasing in subjective risk $v$ because an increase in $v$ raises the risk premium so the principal offers weaker incentives. In our model, however, the effect of an increase in $v$ on incentives depends on whether incentives and employment are complements or substitutes. If $g$ is decreasing in $n$ they are substitutes and the direct effect of an increase in $v$ is to reduce the optimal incentive (28). The indirect effect, however, is to increase the optimal incentive through a reduction in employment. It follows that changes in $v$ have a smaller impact on the optimal incentive than in the standard model when incentives and employment are substitutes. The following example shows that these two effects, direct and indirect, can exactly offset one another so that the optimal incentive is independent of subjective risk.

Example 4 If $\phi \equiv 1, c(n)=n$, and $p^{2}>2 k$ then

$$
n_{b}=\frac{1}{2 v}\left(\frac{\sqrt{2} p}{\sqrt{k}}-2\right) \quad \text { and } \quad \beta_{b}=\sqrt{2 k}
$$

Although it seems unlikely, we cannot rule out the possibility that incentives are increasing in subjective risk over some range which is why we do not report a comparative statics result for $v$ in (ii). Likewise, we cannot make any comparative statics predictions with respect to $p$.

Following Holmström (1982), we now compare the firm with the EDP which will reveal the role of the budget-breaker in our model.

\section{Example 5}

(i) If $\phi(n)=n^{1 / 2}$ and $c(n)=(1 / 4) n^{4}$ there is a unique closed-form solution

$$
n_{b}=\frac{p}{[k(1+v)]^{1 / 2}}, \quad \beta_{b}=\frac{p}{1+v}, \quad \text { and } \quad e_{b}=\left[\frac{p^{6}}{k(1+v)^{5}}\right]^{1 / 4}
$$


to the budget-breaker's problem ignoring the integer constraint on employment.

(ii) In comparison with the EDP in Example 2:

$$
\begin{aligned}
& n_{b} \gtreqless n_{p} \Longleftrightarrow p^{2} \gtreqless(2 / 3) k(1+v)^{3} \\
& \beta_{b} \gtreqless \frac{p}{n_{p}} \Longleftrightarrow p^{2} \gtreqless(3 / 2) k(1+v)^{3} \\
& e_{b} \gtreqless e_{p} \Longleftrightarrow p^{2} \gtreqless(3 / 2)^{1 / 3} k(1+v)^{3} .
\end{aligned}
$$

We chose this example for two reasons. First, it occurs at the boundary between complements and substitutes since $g$ and hence the optimal incentive $\beta_{b}$ are independent of employment $n$. Indeed, $\beta_{b}$ is the same as (37) in the standard model with one agent. We therefore have reason to believe that the results will continue to hold when incentives and employment are weak complements or substitutes. Second, this example is very simple analytically.

For intuitive purposes we compare the expected payoff of the representative partner in (15) and the expected profit of the budget-breaker in (26). First, the $1 / n$ problem with respect to revenue discourages both effort and employment in an EDP as discussed previously. The second set of considerations focus on risk-bearing. While the budget-breaker considers the aggregate risk premium, the representative partner in an EDP only considers his own individual risk premium. Furthermore, the only way that an EDP can reduce the risk premium of the representative partner is to increase employment whereas the budget-breaker can reduce incentives or employment. Since incentives enter the aggregate risk premium in quadratic fashion while employment does so linearly, the former are generally the more effective instrument. Altogether these factors combine to create a major difference between these two institutions: optimal EDP employment $n_{p}$ is increasing in subjective risk $v$ in Proposition 2 whereas $n_{b}$ is decreasing in $v$ in Theorem 1 . Third and finally, the budget-breaker considers the full transaction costs of employment in (26) whereas the representative partner only considers his individual share in (15).

When

$$
p^{2}>(3 / 2) k(1+v)^{3}
$$

the price $p$ is large relative to the transaction cost parameter $k$ and subjective risk $v$ and the $1 / n$ problem with respect to revenue dominates. The EDP is therefore smaller than the firm $n_{p}<n_{b}$ as in Alchian and Demsetz (1972). Since $v$ is relatively low in (43) the firm also offers stronger incentives $p / n_{p}<\beta_{b}$. This matches the role of the budget-breaker in Holmström (1982) except that in our model the budget-breaker's takeover of the EDP would also be accompanied by an 
increase in employment. The combination of stronger incentives and greater sensitivity of effort to incentives implies higher effort in the firm compared to the EDP $e_{p}<e_{b}$ which also matches the classical characterization of the partnership in terms of shirking.

We obtain the opposite characterization when

$$
(8 / 27) k(1+v)^{3}<p^{2}<(2 / 3) k(1+v)^{3},
$$

where the first inequality ensures the EDP operates $U^{p}>0$ (see Example 2). In the second inequality $p$ is low relative to $k$ and $v$ so the primary considerations of the representative partner are the second and third: to reduce his risk premium and the fact that he does not bear the full transaction costs of employment. As a result the EDP is larger than the firm $n_{p}>n_{b}$. Since subjective risk $v$ is relatively high the budget-breaker offers weaker incentives $\beta_{b}<p / n_{p}$ which imply lower effort $e_{b}<e_{p}$. In this case the budget-breaker is more concerned about high transaction costs than low effort and the role of the budget-breaker is to downsize the EDP and offer weaker incentives to reduce risk. This is consistent with the stylized fact that incentives are weak in firms (see Prendergast (1999)). The role of the budget-breaker therefore depends on the economic environment. All of these results survive the imposition of the integer constraint for employment.

We are unaware of any other model in the literature that predicts that a partnership can be larger than a capitalist firm under the same parameter values. In their preliminary analysis of a comprehensive data set of Portuguese firms, Monteiro and Stewart (2012) note that the average size of a labor-managed firm is 22 employees, versus only 10 for the average capitalist firm (excluding sole proprietorships which tend to be even smaller). Moreover, labor-managed firms can be fairly large: up to 800 employees in their data set and as many as 3,000 in others. According to Venn (2009), Portugal is one of the strictest OECD countries in terms of difficulty of dismissal and regulation of temporary contracts which is consistent with the above example where an EDP is larger than the firm when the transaction cost parameter $k$ is sufficiently high.

One of Smith's main contentions in the Wealth of Nations is that specialization and division of labor are the primary sources of national prosperity. In contrast, a Marxist literature initiated by Braverman (1974) alleges that capitalists use machinery and the division of labor to reduce wages and achieve greater control over the production process at the expense of unions or skilled craftsmen. In our model, however, an increase in employment corresponds to greater specialization and division of labor which in turn leads to higher expected wages. 
Theorem 2 If $\phi(n)^{2} g(n, v)$ is strictly increasing in $n$ the expected wage

$$
w_{b}=\alpha_{i}+\beta_{i} \phi(n) \sum_{i} e_{i}
$$

is strictly increasing in $n$ and strongly increasing in $p, k^{-1}$, and $v^{-1}$.

The expected wage is therefore increasing in firm size (as measured by employment) as well as the parameters $\left(p, k^{-1}, v^{-1}\right)$. For example, labor market reforms that reduce transaction costs should also increase wages as well as employment.

\section{Empirical Implementation and Evidence}

We now discuss how the predictions of the model can be tested using cross-sectional data under certain additional assumptions. This discussion relies heavily on Holmström and Milgrom (1994). We begin with some definitions. A vector $x$ of random variables is associated if $\operatorname{Cov}[\gamma(x), \eta(x)] \geq 0$ for all increasing real-valued functions $\gamma$ and $\eta$. If we take $\gamma$ and $\eta$ to be the relevant projection maps we obtain $\operatorname{Cov}(x) \geq 0$ (i.e., all entries of the variance-covariance matrix are nonnegative). If $x$ is associated conditional on any event which is a sublattice then $x$ is affiliated.

Now consider a theoretical model with a vector $y$ of endogenous variables and a vector $x$ of exogenous random parameters. After solving the model we obtain the random variables $(y(x), x)$. If $x=\left(x_{1}, x_{2}\right)$ and $y\left(x_{1}, x_{2}\right)$ is increasing in the subvector $x_{1}$ (but not necessarily in the subvector $\left.x_{2}\right)$ and $x$ is affiliated then $\left(y(x), x_{1}\right)$ is associated conditional on $x_{2}$ fixed at $x_{2}=\bar{x}_{2}$. An empirical analysis of cross-sectional data should therefore find nonnegative correlations between the random variables $\left(y(x), x_{1}\right)$ after controlling for $x_{2}$. Not all of the $x_{1}$ variables need to be observable: if $\tilde{x}_{1}$ is a subvector of $x_{1}$ then $\left(y(x), \tilde{x}_{1}\right)$ is associated conditional on $x_{2}$ fixed at $x_{2}=\bar{x}_{2}$. Finally, the results still hold if the parameters $\tilde{x}_{1}$ are observed with independent errors.

To apply these results we assume $n_{b}$ is a function rather than a correspondence (i.e., we assume a unique optimal employment level for all possible values of the parameters) and that the vector $x=\left(p, k^{-1}, v^{-1}\right)$ of exogenous parameters is affiliated. Note that the latter allows for independence as well as nonnegative correlations among the parameters. We first consider Corollary 1(i), where the endogenous variables $y=\left(\beta_{b}, e_{b}, n_{b}\right)$ are increasing in all the parameters $x$ so we should find nonnegative correlations among $\left(\beta_{b}, e_{b}, n_{b}, p, k^{-1}, v^{-1}\right)$ in cross-sectional data. In particular, we should observe a nonnegative correlation between incentives and employment even if we cannot observe some of the other variables. From Theorems 1 and 2 employment $n_{b}$ and the expected 
wage $w_{b}$ are both increasing in all the parameters $x$ so there should be nonnegative correlations among $\left(n_{b}, w_{b}, p, k^{-1}, v^{-1}\right)$. Note that the prediction that wages are increasing in firm size does not depend on whether incentives and employment are complements or substitutes. In contrast, testing the predictions in Corollary 1(ii) is more difficult. In this case the endogenous variables $\left(\beta_{b}^{-1}, e_{b}, n_{b}\right)$ are increasing in $x_{1}=k^{-1}$ but not necessarily $x_{2}=\left(p, v^{-1}\right)$, so $\left(\beta_{b}^{-1}, e_{b}, n_{b}, k^{-1}\right)$ is associated conditional on $x_{2}=\left(p, v^{-1}\right)$ held constant. In other words, the model only predicts a nonpositive correlation between incentives and employment when price $p$ and subjective risk $v$ are controlled for but these variables (especially $v$ ) are often unobservable.

In summary, the model predicts that when $\phi^{2} / n$ is increasing in $n$ incentives and employment are complements. This is the case when the effects of specialization and division of labor outweigh the cost in terms of idiosyncratic risk. If $\phi^{2} / n$ is decreasing in $n$ then incentives and employment are substitutes and we should observe a weak risk-reward tradeoff (possibly non-existent as in Example 4). Regardless, wages should be increasing in firm size.

The evidence on the relationship between incentives and employment is sketchy, but consistent with the hypothesis that they are substitutes. Rasmusen and Zenger (1990, Lemma 1) show that smaller firms are statistically more efficient at detecting shirking. This leads to the prediction that small firms will put more weight on performance for compensation purposes while large firms will emphasize more easily observed characteristics such as tenure. Empirically, they find that tenure and wages are positively related with a stronger relationship in large firms. Moreover, regressions of weekly earnings on tenure, outside experience, and education have a larger residual variance for small firms, which is consistent with the hypothesis that they weight performance more heavily. Brown and Medoff (1989, p. 1054) find that the standard deviation of wages is smaller in large firms after controlling for the wider range of occupations. If large firms offered stronger incentives we would expect to observe greater wage variation than in small firms. Schaefer (1998) considers a version of the standard linear model where risk increases with firm size in a simple reduced-form way and shows that incentives therefore decrease with firm size. Using data on executive teams he finds support for this prediction although size is measured by assets or market value of the firm rather than employment.

In his influential survey, Prendergast (1999) reviews the empirical literature on the risk-reward tradeoff and concludes that the evidence is "rather mixed" with "some studies showing the effect of noise on piece rates while others show little." This conclusion is consistent with the above evidence that incentives and employment are substitutes. There is, however, an alternative interpretation: if the extent and effects of specialization and division of labor vary across different production 
processes then this may help explain the mixed nature of the results. We should also point out that much of the aforementioned evidence is based on CEO compensation which may not be appropriate for testing the predictions of the present model.

The prediction that wages are increasing in firm size has broad and consistent empirical support; see Brown and Medoff (1989). As discussed in that paper, conventional explanations include: larger firms employ better workers, pay a compensating differential because of inferior fringe benefits or working conditions, and do not monitor their employees as effectively, as well as the hypotheses of union avoidance and market power. Brown and Medoff find little or no evidence in support of any of these explanations except worker quality, which only explains about half of the size-wage differential. In our model the participation constraint binds so the expected wage is equal to the cost of effort plus the individual risk premium. The expected wage is therefore increasing in employment because agents exert higher effort and/or bear more risk in larger firms.

\section{Conclusion}

In this paper we developed a model of incentives, wages, employment, and the division of labor in teams. The main contributions are twofold. First, the paper establishes interdependencies between the employment decision and incentive contracting. We provided conditions under which incentives and employment are complements or substitutes. We showed how the strength of the risk-reward tradeoff depends on the relationship between incentives and employment and how wages depend on firm size. Finally, our comparative statics results demonstrate how labor market parameters can influence incentive contracting and parameters traditionally emphasized in the moral hazard literature (e.g., subjective risk) can affect the demand for labor. These results can explain evidence that incentives and employment are substitutes which cannot be addressed satisfactorily in models where employment is exogenous. It also provides new explanations for phenomena such as the empirically weak risk-reward tradeoff and the size-wage differential. Most importantly, it can explain all these empirical regularities within a single theoretical framework.

The second main contribution of the paper is to provide a richer characterization of the partnership and the role of Holmström's budget-breaker. In environments where subjective risk and transaction costs are low (e.g., a modern economy with liberalized labor markets) we obtain the classical characterization of the partnership as small with weak incentives and low effort. In this case the role of the budget-breaker is to increase employment and strengthen incentives. But in

environments where subjective risk and transaction costs are high (e.g., a modern economy with 
relatively inflexible labor markets or a pre-industrial mercantile economy) we obtain the opposite characterization and the role of the budget-breaker is more Knightian in essence: to weaken incentives and reduce the agents' exposure to risk and to decrease employment to save on transaction costs.

\section{Appendix}

Derivation of Equation 2. We show that the production function in (2) can be derived in a framework similar to that in Becker and Murphy (1992). Consider a production process with a continuum $S=[0,1]$ of tasks, where $S_{i} \subseteq S$ is the set of tasks assigned to agent $i$. We assume that for practical reasons (discussed later) the principal assigns the same proportion of tasks to each agent so the Lebesgue measure of $S_{i}$ is $\rho_{i}=1 / n$. Let $q_{i s}=\Gamma\left(l_{i s}\right) e_{i s}$ be the output of agent $i$ in task $s \in S_{i}$, where $\Gamma$ is strictly increasing, $l_{i s}$ is the time spent learning about task $s$, and $e_{i s}$ is actual production effort. For simplicity we assume each agent has a fixed allocation $l$ of time that can be devoted to learning but can supply a variable total effort $e_{i}$ at $\operatorname{cost}(1 / 2) e_{i}^{2}$, where

$$
e_{i}=\int_{S_{i}} e_{i s} d s
$$

Recall that in Becker and Murphy each agent supplies one unit of effort inelastically; in this paper we allow for an elastic supply $e_{i}$ of effort to allow for moral hazard. The total output of agent $i$ is

$$
Q_{i}=\int_{S_{i}} q_{i s} d s+\epsilon_{i}
$$

where $\epsilon_{i}$ is an i.i.d. normally distributed idiosyncratic shock with mean zero and variance $\sigma^{2}$. Team output is given by $Q=\sum_{i} Q_{i}$.

Given fixed values for $l$ and $e_{i}$ we now consider the agent's optimal allocation of $l$ and $e_{i}$ across tasks; i.e., we consider the problem of choosing $e_{i s}$ and $l_{i s}$ to maximize expected output

$$
\int_{S_{i}} \Gamma\left(l_{i s}\right) e_{i s} d s
$$

subject to $e_{i s}, l_{i s} \geq 0,(\mathrm{~A} 1)$, and

$$
l=\int_{S_{i}} l_{i s} d s
$$

Since $\Gamma$ is strictly increasing the solution to this isoperimetric problem (see Kamien and Schwartz (1981, p. 43-47)) is $e_{i s}=e_{i} / \rho_{i}=n e_{i}$ and $l_{i s}=l / \rho_{i}=n l$ for all $s \in S_{i}$ because $\rho_{i}=1 / n$ for all $i$. It 
follows that

$$
Q_{i}=\Gamma\left(l / \rho_{i}\right) e_{i}+\epsilon_{i}=\Gamma(n l) e_{i}+\epsilon_{i}
$$

and

$$
Q=\phi(n) \sum_{i} e_{i}+\epsilon
$$

where $\phi(n) \equiv \Gamma(n l)$ and $\epsilon=\sum_{i} \epsilon_{i}$ is normally distributed with mean zero and variance $n \sigma^{2}$. Note that the expected output of agent $i$ is decreasing in the proportion $\rho_{i}$ of tasks assigned to $i$ because more tasks implies less learning per task.

In the working paper version of this paper we allow $\left\{\rho_{i}\right\}$ to be endogenous and chosen by the principal. In general, the principal has an incentive to set $\rho_{j}$ close to one for some agent $j$ and $\rho_{i}$ close to zero for all $i \neq j$ because $e_{i s}=e_{i} / \rho_{i}$ and $l_{i s}=l / \rho_{i}$ explode as $\rho_{i} \rightarrow 0$. As in Becker and Murphy, in the working paper version we circumvent this problem by assuming a Leontief production function where efforts are perfect complements which makes it optimal for the principal to set $\rho_{i}=1 / n$ for all $i$. In the present paper we assume an equal division of tasks because otherwise the production process would be slowed not only because some agents have more tasks to do than others but also because the agents with more tasks would be further slowed by their relative lack of specialization.

Proof of Proposition 1. If we can show that (ii) holds then (iii) follows from an application of theorem 2.3 in Vives (1999). Let $\left(p_{1}, k_{1}^{-1}\right)>\left(p_{2}, k_{2}^{-1}\right)$, which implies $p_{1} \geq p_{2}$ and $k_{2} \geq k_{1}$ with at least one strict. Since

$$
V\left(n, p_{1}, k_{1}^{-1}\right)-V\left(n, p_{2}, k_{2}^{-1}\right)=(1 / 2) n \phi(n)^{2}\left(p_{1}^{2}-p_{2}^{2}\right)+\left[\left(k_{2}^{-1}\right)^{-1}-\left(k_{1}^{-1}\right)^{-1}\right] c(n)
$$

is strictly increasing in $n$, we are done.

Proof of Proposition 2. If $\left(p_{1}, k_{1}^{-1}, v_{1}\right)>\left(p_{2}, k_{2}^{-1}, v_{2}\right)$ then

$$
U^{p}\left(n, p_{1}, k_{1}^{-1}, v_{1}\right)-U^{p}\left(n, p_{2}, k_{2}^{-1}, v_{2}\right)=\left(p_{1}^{2}-p_{2}^{2}\right) f(n)-\frac{1}{2 n}\left(p_{1}^{2} v_{1}-p_{2}^{2} v_{2}\right)+\left(k_{2}-k_{1}\right) \frac{c(n)}{n}
$$

is strictly increasing in $n$.

Proof of Theorem 1. Let $X \subseteq \mathbb{R}^{m}$ and $T \subseteq \mathbb{R}^{l}$. Recall from Topkis (1998, p. 42) that $f: X \times T \rightarrow \mathbb{R}$ has strictly increasing differences in $x=\left(x_{1}, \ldots, x_{m}\right)$ and $t=\left(t_{1}, \ldots, t_{l}\right)$ iff $f$ has strictly increasing differences in all pairs $\left(x_{i}, t_{j}\right)$ with the rest of $x$ and $t$ held constant. If 
$\left(p_{1}, k_{1}^{-1}\right)>\left(p_{2}, k_{2}^{-1}\right)$ then

$$
\begin{aligned}
& \Pi\left(n, p_{1}, k_{1}, v\right)-\Pi\left(n, p_{2}, k_{2}, v\right)= \\
& \left(p_{1}^{2}-p_{2}^{2}\right)(1 / 2) n \phi(n)^{2} g(n, v)+\left(k_{2}-k_{1}\right) c(n)
\end{aligned}
$$

increases as $n$ increases from 0 to 1 because $\phi(1)>0$ and $g(n, v)>0$ for all $n \geq 1$. It is strictly increasing in $n \geq 1$ because

$$
n \phi(n)^{2} g(n, v)=\frac{\phi(n)^{2}}{\frac{1}{n}+\frac{v}{\phi(n)^{2}}} .
$$

It follows that $\Pi$ has strictly increasing differences in $n$ and $\left(p, k^{-1}\right)$. If $v_{1}^{-1}>v_{2}^{-1}$ then

$$
\begin{aligned}
\Pi\left(n, p, k, v_{1}\right)-\Pi\left(n, p, k, v_{2}\right) & =(1 / 2) p^{2} n \phi(n)^{2}\left[g\left(n, v_{1}\right)-g\left(n, v_{2}\right)\right] \\
& =\frac{p^{2}\left(v_{2}-v_{1}\right)}{2\left(\frac{1}{n}+\frac{v_{1}}{\phi(n)^{2}}\right)\left(\frac{1}{n}+\frac{v_{2}}{\phi(n)^{2}}\right)},
\end{aligned}
$$

is strictly increasing in $n \geq 0$. It follows that $\Pi$ has strictly increasing differences in $n$ and $v^{-1}$. Combining this result with the previous one, $\Pi$ has strictly increasing differences in $n$ and $\left(p, k^{-1}, v^{-1}\right)$, which completes the proof.

Proof of Theorem 2. Using the participation constraint,

$$
\alpha_{i}+\beta_{i} \phi(n) \sum_{i} e_{i}=(1 / 2) e_{i}^{2}+(1 / 2) n v \beta_{i}^{2}
$$

Substituting optimal effort (31) and the optimal incentive (28),

$$
\alpha_{i}+\beta_{i} \phi(n) \sum_{i} e_{i}=(1 / 2) p^{2} \phi(n)^{2} g(n, v)
$$

The rest of the result follows from Theorem 1 .

\section{References}

Alchian, A.A., Demsetz, H., 1972. Production, information costs, and economic organization. Amer. Econ. Rev. 62(5) 777-795.

Auriol, E., Friebel, G., Pechlivanos, L. 1999. Teamwork management in an era of diminishing 
commitment. CEPR Discussion Paper \# 2281.

Auriol, E., Friebel, G., Pechlivanos, L. 2002. Career concerns in teams. J. Labor Econ. 20(2) 289-307.

Bartling, B., von Siemens, F.A., 2011. Equal sharing rules in partnerships. J. Instit. Theor. Econ. 166(2) 299-320.

Becker, G.S., Murphy, K.M., 1992. The division of labor, coordination costs, and knowledge. Quart. J. Econ. 107(4) 1137-1160.

Bolton, P., Dewatripont, M., 2005. Contract Theory. MIT Press, Cambridge, Mass.

Bose, A., Pal, D., Sappington, D.E.M., 2011. On the performance of linear contracts. J. Econ. Man. Strat. 20(1) 159-193.

Bose, A., Pal, D., Sappington, D.E.M., 2010. Equal pay for unequal work: limiting sabotage in teams. J. Econ. Man. Strat. 19(1) 25-53.

Braverman, H., 1974. Labor and Monopoly Capital. Monthly Review Press, New York, NY.

Brown, C., Medoff, J., 1989. The employer size-wage effect. J. Polit. Econ. 97(5) 1027-1059.

Carter, N.M., Keon, T.L., The rise and fall of the division of labour, the past 25 years. 1986. Organ. Stud. 7(1) 57-74.

Encinosa, W.E., Gaynor, M., Rebitzer, J.B., 2007. The sociology of groups and the economics of incentives: theory and evidence on compensation systems. J. Econ. Behav. Org. 62(2) $187-214$.

Garicano, L., Santos, T., 2004. Referrals. Amer. Econ. Rev. 94(3) 499-525.

Holmström, B., 1982. Moral hazard in teams. Bell J. Econ. 13(2) 324-340.

Holmström, B., Milgrom, P., 1987. Aggregation and linearity in the provision of intertemporal incentives. Econometrica 55(2) 303-328.

Holmström, B., Milgrom, P., 1991. Multitask principal-agent analyses: incentive contracts, asset ownership, and job design. J. Law Econ. Org. 7(1) 24-52. 
Holmström, B., Milgrom, P., 1994. The firm as an incentive system. Amer. Econ. Rev. 84(4) 972-991.

Itoh, H., 1994. Job design, delegation and cooperation: a principal-agent analysis. Europ. Econ. Rev. 38(3-4) 691-700.

Kamien, M.I., Schwartz, N.L., 1981. Dynamic Optimization: The Calculus of Variations and Optimal Control in Economics and Management. North-Holland, New York, NY.

Lang, K., Gordon, P-J., 1995. Partnerships as insurance devices: theory and evidence. RAND J. Econ. 26(4) 614-629.

Legros, P., Matsushima, H., 1991. Efficiency in partnerships. J. Econ. Theory 55(2) 296-392.

Legros, P., Matthews, S.A., 1993. Efficient and nearly-efficient partnerships. Rev. Econ. Stud. 60(3) 599-611.

Levin, J., Tadelis, S., 2005. Profit-sharing and the role of professional partnerships. Quart. J. Econ. 120(1) 131-171.

Liang, P.J., Rajan, M.V., Ray, K., 2008. Optimal team size and monitoring in organizations. The Acc. Rev. 83(3) 789-822.

Milgrom, P., Roberts, J., 1990. Rationalizability, learning, and equilibrium in games with strategic complementarities. Econometrica 58, 1255-77.

Monteiro, N.P., Stewart, G., 2012. New comparative evidence on labor-managed and capitalist production. Working paper.

Prendergast, C., 1999. The provision of incentives in firms. J. Econ. Lit. 37(1) 7-63.

Rasmusen, E., 1987. Moral hazard in risk averse teams. RAND J. Econ. 18(3) 428-435.

Rasmusen, E., Zenger, T., 1990. Diseconomies of scale in employment contracts. J. Law Econ. Org. 6(1) 65-92.

Schaefer, S., 1998. The dependence of pay-performance sensitivity on the size of the firm. Rev. Econ. Stat. 80(3) 436-443. 
Schmitz, P.W., 2005. Allocating control in agency problems with limited liability and sequential hidden actions. RAND 36(2) 318-336.

Schöttner, A., Relational contracts, multitasking, and job design. J. Law Econ. Org. 24(1) 138-162.

Topkis, D.M., 1998. Supermodularity and Complementarity. Princeton University Press, Princeton.

Venn, D., 2009. Legislation, collective bargaining and enforcement: updating the OECD employment protection indicators. OECD Social, Employment and Migration Working Papers \# 89.

Vives, X., 1999. Oligopoly Pricing. MIT Press, London. 